\title{
Two-Dimensional Toda-Heisenberg Lattice
}

Vadim E. VEKSLERCHIK

Institute for Radiophysics and Electronics of NAS of Ukraine, 12, Proskura Str., Kharkiv, 61085, Ukraine

E-mail:vekslerchik@yahoo.com

Received February 06, 2013, in final form June 04, 2013; Published online June 12, 2013

http://dx.doi.org/10.3842/SIGMA.2013.044

\begin{abstract}
We consider a nonlinear model that is a combination of the anisotropic twodimensional classical Heisenberg and Toda-like lattices. In the framework of the Hirota direct approach, we present the field equations of this model as a bilinear system, which is closely related to the Ablowitz-Ladik hierarchy, and derive its $N$-soliton solutions.
\end{abstract}

Key words: classical Heisenberg model; Toda-like lattices; Hirota direct method; AblowitzLadik hierarchy; soliton

2010 Mathematics Subject Classification: 39A14; 82D40; 35C08; 11C20

\section{Introduction}

In this paper we consider a two-dimensional lattice that can be viewed as a generalization of the anisotropic two-dimensional classical Heisenberg model [4, 18],

$$
\mathcal{E}_{\text {Heis }}=\sum_{\boldsymbol{r} \in \Lambda} \sum_{i=1,2} J_{i}\left(\phi_{\boldsymbol{r}}, \phi_{\boldsymbol{r}+\boldsymbol{\delta}_{i}}\right)
$$

where $\phi_{r}$ is a three-dimensional unit vector,

$$
\left(\phi_{r}, \phi_{r}\right)=1
$$

(with brackets standing for the standard scalar product), $J_{1,2}$ are the constants characterizing the interaction between near-neighbour sites (exchange constants) and $\Lambda$ is a two-dimensional lattice formed by two vectors $\boldsymbol{\delta}_{1}$ and $\boldsymbol{\delta}_{2}$ :

$$
\Lambda=\left\{m_{1} \boldsymbol{\delta}_{1}+m_{2} \boldsymbol{\delta}_{2}\right\}_{m_{1}, m_{2}=0, \pm 1, \pm 2, \ldots} .
$$

The generalization that we are going to study consists in replacing the constants $J_{i}$ with some functions of new variables. In more details, we associate with each site, in addition to the vector $\phi_{r}$, a new variable $u_{\boldsymbol{r}}$ and modify the exchange constants as

$$
J_{i} \rightarrow J_{i} \exp \left\{u_{\boldsymbol{r}}-u_{\boldsymbol{r}+\boldsymbol{\delta}_{i}}\right\} .
$$

An elementary example that leads to the above modification of the exchange interaction is to permit the spins to oscillate in the direction perpendicular to the plane and to state that the interaction coefficients $J$ depend on the distance (in the three-dimensional space) between the spins: $\mathcal{E}_{a b}=J\left(\left|\boldsymbol{R}_{a}-\boldsymbol{R}_{b}\right|\right)\left(\phi_{\boldsymbol{r}_{a}}, \boldsymbol{\phi}_{\boldsymbol{r}_{b}}\right)$, where $\boldsymbol{R}_{a}=\boldsymbol{r}_{a}+u_{\boldsymbol{r}_{a}} \boldsymbol{\nu}$, with $\boldsymbol{\nu} \perp \boldsymbol{\delta}_{1,2}$. In this case the interaction between the nearest neighbours depends on $\left|\boldsymbol{\delta}_{i}\right|$ and $\left|u_{\boldsymbol{r}}-u_{\boldsymbol{r}+\boldsymbol{\delta}_{i}}\right|$, that can be modeled by (1.1). Of course, the dependence given by (1.1) is far from being realistic, however this toy model can give some insight into effects caused by such kind on nonlinearities, and especially into the possibility of appearing of specific structures like solitons that are discussed in this paper. 

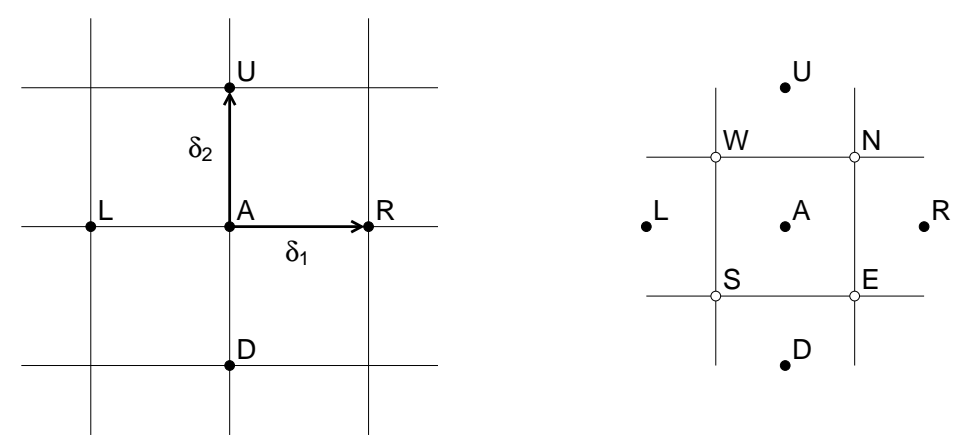

Figure 1. Direct and dual lattices, nearest-neighbour notation.

To summarize, our model is described by the energy functional

$$
\mathcal{E}=\sum_{\boldsymbol{r} \in \Lambda} \sum_{i=1,2} J_{i} \exp \left\{u_{\boldsymbol{r}}-u_{\boldsymbol{r}+\boldsymbol{\delta}_{i}}\right\}\left(\boldsymbol{\phi}_{\boldsymbol{r}}, \boldsymbol{\phi}_{\boldsymbol{r}+\boldsymbol{\delta}_{i}}\right) .
$$

It is easy to see that neglecting the $\phi_{\boldsymbol{r}}$-part, or imposing the restrictions $\left(\boldsymbol{\phi}_{\boldsymbol{r}}, \boldsymbol{\phi}_{\boldsymbol{r}+\boldsymbol{\delta}_{i}}\right)=c_{i}$ for all $\boldsymbol{r}$ and redefining the constants $J_{i}$, one arrives at the one of the Hirota's versions of the discrete 2D Toda lattice [13, 14, 20],

$$
\mathcal{E}_{\text {Toda }}=\sum_{\boldsymbol{r} \in \Lambda} \sum_{i=1,2} J_{i} \exp \left\{u_{\boldsymbol{r}}-u_{\boldsymbol{r}+\boldsymbol{\delta}_{i}}\right\}
$$

whose field equations $\delta \mathcal{E}_{\text {Toda }} / \delta u_{\boldsymbol{r}}=0$ are known to be integrable. Thus, we call model (1.2), which is the subject of this paper, the two-dimensional Toda-Heisenberg lattice (2DTHL).

To make the following formulae more readable we introduce the alternative notation: instead of the vector index we will use a letter one,

$$
\phi_{\boldsymbol{r}}, u_{\boldsymbol{r}} \rightarrow \phi_{A}, u_{A}
$$

and denote the nearest neighbours of the point $A$ as indicated in Fig. 1 ( $R, L, U$ and $D$ stand for 'right', 'left', 'up', 'down'). The energy of the model can be rewritten as

$$
\mathcal{E}=\frac{1}{2} \sum_{A} \mathcal{E}_{A},
$$

where

$$
\mathcal{E}_{A}=\sum_{B} J_{B} \exp \left\{\varepsilon_{B}\left(u_{A}-u_{B}\right)\right\}\left(\phi_{A}, \phi_{B}\right)
$$

and the summation index runs over the nearest neighbours,

$$
\sum_{B} \cdots=\sum_{B=R, U, L, D} \cdots
$$

The constants $J_{B}$ are nothing but $J_{1,2}$,

$$
J_{R}=J_{L}=J_{1}, \quad J_{U}=J_{D}=J_{2}
$$

while $\varepsilon_{B}$ takes into account the signs of $u_{B}$ in the arguments of the exponential functions,

$$
\varepsilon_{R}=-\varepsilon_{L}=\varepsilon_{U}=-\varepsilon_{D}=1
$$


Table 1. Nearest-neighbour notation and parameters.

\begin{tabular}{|c|c|c|c|c|c|c|}
\hline$B$ & $B^{\prime}$ & $B^{\prime \prime}$ & $J_{B}$ & $\varepsilon_{B}$ & $\xi_{B}$ & $\mu_{B}$ \\
\hline$R$ & $E$ & $N$ & $J_{1}$ & +1 & $\xi_{1}$ & $\mu$ \\
$U$ & $N$ & $W$ & $J_{2}$ & +1 & $\xi_{2}$ & $-1 / \mu$ \\
$L$ & $W$ & $S$ & $J_{1}$ & -1 & $\xi_{1}$ & $\mu$ \\
$D$ & $S$ & $E$ & $J_{2}$ & -1 & $\xi_{2}$ & $-1 / \mu$ \\
\hline
\end{tabular}

(see Table 1). The central object of the presented study are the Euler-Lagrange equations corresponding to (1.4) with (1.5),

$$
\begin{aligned}
& \frac{\delta \mathcal{E}_{A}}{\delta u_{A}}=\sum_{B} \varepsilon_{B} J_{B} \exp \left\{\varepsilon_{B}\left(u_{A}-u_{B}\right)\right\}\left(\phi_{A}, \phi_{B}\right)=0, \\
& \frac{\delta \mathcal{E}_{A}}{\delta \phi_{A}}=\sum_{B} J_{B} \exp \left\{\varepsilon_{B}\left(u_{A}-u_{B}\right)\right\}\left[\phi_{A} \times \phi_{B}\right]=0 .
\end{aligned}
$$

Here, we have calculated the derivative with respect to $\phi_{A}$ bearing in mind the fact that $\left|\phi_{A}\right|=1$, which implies that the admissible variations should be orthogonal to $\phi_{A}$, which in its turn leads to the 'definition' $\delta\left(\phi_{A}, \boldsymbol{\psi}\right) / \delta \phi_{A}=\left[\phi_{A}, \boldsymbol{\psi}\right]$. The same result, (1.7), can be reproduced by means of the Lagrange multipliers.

\section{Bilinearization of the 2DTHL}

In this section we bilinearize the field equations (1.6) and (1.7). This will be done in several steps. First we replace the vector variables $\phi_{A}$ with scalar ones using a parametrization which can be viewed as an alternative to the stereographic projection. Secondly, we reduce some of the fivesite equations (star-equations) with simpler ones (quad-equations). Then, we introduce the taufunctions and, finally, split (in the next section) the obtained bilinear equations into the standard three-term Hirota-like ones, which are closely related to the Ablowitz-Ladik hierarchy (ALH).

\subsection{Scalar equations}

It is easy to check that any three-dimensional unit vector $\phi, \phi^{2}=1$, can be presented in terms of a single complex function $q$ as

$$
\phi=\frac{1}{\sqrt{p}}\left(\begin{array}{c}
\operatorname{Re} q \\
\operatorname{Im} q \\
1
\end{array}\right)
$$

where

$$
p=1+|q|^{2} \text {. }
$$

In terms of $q$ the scalar and vector products are given by

$$
\begin{aligned}
& \left(\phi_{A}, \phi_{B}\right)=\frac{1}{\sqrt{p_{A} p_{B}}}\left(1+\operatorname{Re} q_{A} q_{B}^{*}\right), \\
& {\left[\phi_{A} \times \phi_{B}\right]=\frac{1}{\sqrt{p_{A} p_{B}}} \operatorname{Im}\left(q_{A}-q_{B}\right)\left(\begin{array}{c}
1 \\
-i \\
-q_{A}^{*}
\end{array}\right)}
\end{aligned}
$$


(the asterisk denotes the complex conjugation). Using these formulae and replacing $q_{A}^{*}$ with the additional variable $r_{A}$,

$$
r_{A}=-q_{A}^{*}
$$

one can rewrite the field equations (1.6) and (1.7) as follows

$$
\begin{aligned}
0 & =\sum_{B} f_{A B}\left(q_{A}-q_{B}\right), \\
0 & =\sum_{B} f_{A B}\left(r_{A}-r_{B}\right), \\
0 & =\sum_{B} \varepsilon_{B} f_{A B}\left[1-\frac{1}{2}\left(q_{A} r_{B}+q_{B} r_{A}\right)\right],
\end{aligned}
$$

where

$$
f_{A B}=J_{B} \frac{\exp \left\{\varepsilon_{B}\left(u_{A}-u_{B}\right)\right\}}{\sqrt{p_{A} p_{B}}} .
$$

\subsection{Quad-equations}

Now we arrive at the key moment of bilinearization of our equations. It consists in introducing the dual lattice, as is shown in Fig. 1, whose nodes closest to the point $A$ are denoted by the letters $N, W, S$ and $E$ (coming from 'north', 'west', 'south', and 'east'), and extending the functions $q_{A}$ and $r_{A}$ to the points of the dual lattice by

$$
\begin{aligned}
& f_{A B}\left(q_{A}-q_{B}\right)=c\left(q_{B^{\prime}}-q_{B^{\prime \prime}}\right), \\
& f_{A B}\left(r_{A}-r_{B}\right)=c\left(r_{B^{\prime}}-r_{B^{\prime \prime}}\right),
\end{aligned}
$$

where $B^{\prime}$ and $B^{\prime \prime}$ are the ends of the oriented edge of the dual lattice that crosses the edge $(A B)$ of the direct one (see Table 1) and $c$ is a constant. Of course, equations (2.6), (2.7) cannot be viewed as definitions of $q_{B^{\prime}}$ and $r_{B^{\prime}}$ because the determinants of the right-hand sides is zero. We propose them as just an ansatz. Its role is that it 'solves' 4-star equations (2.3) and (2.4). Indeed, the right-hand sides of (2.3) and (2.4) are now given by

$$
\text { r.h.s. }(2.3)=c \sum_{B}\left(q_{B^{\prime}}-q_{B^{\prime \prime}}\right), \quad \text { r.h.s. }(2.4)=c \sum_{B}\left(r_{B^{\prime}}-r_{B^{\prime \prime}}\right)
$$

and are identically zero because of the cyclic character of $B^{\prime}$ and $B^{\prime \prime}$ (see Table 1 ). However, substitutions (2.6) and (2.7) are not so easy as it may appear. The problem lies in the fact that the consistency of equations (2.6) and (2.7) with the lattice translations implies some restrictions on the functions $f_{A B}$. To expose them, let us apply to (2.6) with $B=U$ the shift in the 'east' direction, $\mathbb{T}_{E}$, defined by

$$
\mathbb{T}_{E} f_{A}=f_{E}, \quad \mathbb{T}_{E} f_{W}=f_{A}, \quad \mathbb{T}_{E} f_{L}=f_{S}, \quad \text { etc. }
$$

By simple algebra one can obtain that functions $f_{A B}$ have to meet the condition

$$
f_{A R} \mathbb{T}_{E} f_{A U}=-c^{2},
$$

which in terms of $u$ and $p$ is given by

$$
J_{1} J_{2} \exp \left(u_{A}-u_{R}+u_{E}-u_{N}\right)=-c^{2} \sqrt{p_{A} p_{R} p_{E} p_{N}}-
$$

Thus, we have replaced equations (2.3) and (2.4) with new ones, (2.6) and (2.7), together with (2.9). 


\subsection{Rebuilding (2.5)}

Ansatz (2.6) and (2.7) not only enables to 'solve' equation (2.3) and (2.4) but also gives us possibility to simplify the remaining field equation (2.5).

With the help of (2.6) and (2.7) one can present the right-hand side of (2.5) as

$$
\text { r.h.s. }(2.5)=\mathcal{X}_{A}+\mathcal{Y}_{A}
$$

with

$$
\mathcal{X}_{A}=\frac{c}{2} \sum_{B} \varepsilon_{B}\left(\{q, r\}_{A B^{\prime}}-\{q, r\}_{A B^{\prime \prime}}\right), \quad \mathcal{Y}_{A}=p_{A} \sum_{B} f_{A B} \varepsilon_{B},
$$

where we use the shorthand

$$
\{x, y\}_{A B}=x_{A} y_{B}+x_{B} y_{A}
$$

The first part, $\mathcal{X}_{A}$, after substituting $\varepsilon_{B}$, becomes

$$
\mathcal{X}_{A}=c\left(\{q, r\}_{A E}-\{q, r\}_{A W}\right)=c\left(\mathbb{T}_{E}-1\right)\{q, r\}_{A W} .
$$

The last expression indicates that there is a possibility to reduce the order of our equation by presenting the right-hand side of $(2.5)$ as $\left(\mathbb{T}_{E}-1\right) \mathcal{S}_{A}$ and solving $\mathcal{S}_{A}=$ const. To do this, we rewrite $\mathcal{Y}_{A}$ with the help of $(2.8)$ as

$$
\mathcal{Y}_{A}=p_{A}\left(f_{A U}-f_{A L}\right)+c^{2} \mathbb{T}_{E} p_{W}\left(f_{A L}^{-1}-f_{A U}^{-1}\right)
$$

and note that we can achieve our goal by imposing the condition

$$
p_{A} f_{A U} f_{A L}=-c^{2} p_{W}
$$

This equation, after substituting $f_{A B}$ and applying $\mathbb{T}_{E}$, becomes

$$
J_{1} J_{2} \exp \left(u_{S}-u_{N}\right)=-c^{2} p_{A} \sqrt{p_{S} p_{N}}
$$

and converts (2.5) into

$$
c\{q, r\}_{A W}+p_{A}\left(f_{A L}-f_{A U}\right)=\lambda=\text { const. }
$$

To summarize, at this stage the field equations can be written as the system

$$
\begin{aligned}
& f_{A L}\left(q_{A}-q_{L}\right)=c\left(q_{W}-q_{S}\right), \quad f_{A L}\left(r_{A}-r_{L}\right)=c\left(r_{W}-r_{S}\right), \\
& c\{q, r\}_{A W}+p_{A}\left(f_{A L}-f_{A U}\right)=\lambda
\end{aligned}
$$

(here we write equations (2.6) and (2.7) with $B=L$, keeping in mind that all the rest can be obtained by lattice shifts) together with

$$
\begin{aligned}
& J_{1} J_{2} \exp \left(u_{L}-u_{A}+u_{S}-u_{W}\right)=-c^{2} \sqrt{p_{L} p_{A} p_{S} p_{W}} \\
& J_{1} J_{2} \exp \left(u_{S}-u_{N}\right)=-c^{2} p_{A} \sqrt{p_{S} p_{N}} .
\end{aligned}
$$




\subsection{Tau-functions}

It turns out that one can solve equations (2.11) explicitly, by introducing proper parametrization of the functions $u$ and $p$. Omitting the technical details, we present here the results.

By easy calculation one can check that functions

$$
u_{A}=w_{A}+\frac{1}{2} \ln \frac{\tau_{S}}{\tau_{N}}, \quad p_{A}=\frac{\tau_{N} \tau_{S}}{\tau_{A}^{2}},
$$

where $w$ is a linear function of $\boldsymbol{r}$ solve (2.11) identically provided that $w$ satisfies

$$
J_{1} J_{2} \exp \left(w_{S}-w_{N}\right)=-c^{2} .
$$

The role of the function $w$, which in terms of $\boldsymbol{r}$ can be written as $w=(\boldsymbol{k}, \boldsymbol{r})$ with a constant vector $\boldsymbol{k}$, is that it describes (in the case of bounded tau-functions $\tau$ ) the asymptotics of solutions:

$$
u \sim(\boldsymbol{k}, \boldsymbol{r}) \quad \text { as } \quad|\boldsymbol{r}| \rightarrow \infty .
$$

Substituting $u_{A}$ and $p_{A}$ one can rewrite $f_{A B}$ as

$$
f_{A B}=J_{B} \xi_{B} \frac{\tau_{A} \tau_{B}}{\tau_{B^{\prime}} \tau_{B^{\prime \prime}}} .
$$

Here $J_{B}$ and $\xi_{B}$ are given in Table 1 , the constants $\xi_{1,2}$ are defined by

$$
\xi_{i}=\exp \left\{-\left(\boldsymbol{k}, \boldsymbol{\delta}_{i}\right)\right\}, \quad i=1,2,
$$

and are related by

$$
J_{1} J_{2} \xi_{1} \xi_{2}=-c^{2},
$$

which ensures that (2.13) is met.

The structure of equations (2.6), (2.7) and (2.15) suggests the representation of $q_{A}$ and $r_{A}$,

$$
q_{A}=\frac{\sigma_{A}}{\tau_{A}}, \quad r_{A}=\frac{\rho_{A}}{\tau_{A}},
$$

where real tau-functions $\tau$ and complex ones, $\sigma$ and $\rho$, are related by

$$
\tau_{A}^{2}=\sigma_{A} \rho_{A}+\tau_{N} \tau_{S}
$$

which is the consequence of the identity $p_{A}=1-q_{A} r_{A}$. This representation immediately converts (2.6) and (2.7) into bilinear equations

$$
0=[\sigma, \tau]_{A B}-\mu_{B}[\sigma, \tau]_{B^{\prime} B^{\prime \prime}}, \quad 0=[\rho, \tau]_{A B}-\mu_{B}[\rho, \tau]_{B^{\prime} B^{\prime \prime}},
$$

where we utilize another shorthand,

$$
[x, y]_{A B}=x_{A} y_{B}-x_{B} y_{A},
$$

and use, instead of $J_{B}$ and $\xi_{B}$, constants $\mu_{B}$ collected in Table 1, with

$$
\mu=\frac{c}{J_{1} \xi_{1}}=-\frac{J_{2} \xi_{2}}{c} .
$$

Finally, one can see that the right-hand side of the last field equation, (2.10), becomes

$$
\text { r.h.s. }(2.10)=\frac{c}{\tau_{A} \tau_{W}}\left(\{\sigma, \rho\}_{A W}+\mu \tau_{U} \tau_{S}+\mu^{-1} \tau_{N} \tau_{L}\right)
$$


that leads to the bilinearization of $(2.10)$ :

$$
\{\sigma, \rho\}_{A W}+\mu \tau_{U} \tau_{S}+\mu^{-1} \tau_{L} \tau_{N}=\frac{\lambda}{c} \tau_{A} \tau_{W} .
$$

In what follows we restrict ourselves to the case of bounded $u-w$, which corresponds to

$$
\lambda=c\left(\mu+\mu^{-1}\right) .
$$

Thus, we have proved the following

Proposition 2.1. A wide range of solutions for the field equations (1.6) and (1.7) can be obtained from the bilinear system

$$
\begin{aligned}
& 0=\tau_{A}^{2}-\tau_{N} \tau_{S}-\sigma_{A} \rho_{A}, \\
& 0=[\sigma, \tau]_{A L}+\mu[\sigma, \tau]_{S W} \\
& 0=[\rho, \tau]_{A L}+\mu[\rho, \tau]_{S W} \\
& 0=\{\sigma, \rho\}_{A W}+\mu \tau_{U} \tau_{S}+\mu^{-1} \tau_{L} \tau_{N}-\left(\mu+\mu^{-1}\right) \tau_{A} \tau_{W} .
\end{aligned}
$$

This system is the one we were looking for: a bilinear system providing solutions for the field equations (1.6) and (1.7). However, these equations, except the first one, are four- and five-site ones, which makes their solution rather cumbersome. It is possible to prove directly that solutions presented below (see section 4) satisfy (2.17). However we take another way and split, in the next section, this system in a set of standard Hirota-like three-term equations. Of course, such splitting narrows the class of solutions. Nevertheless, this class is rather rich and contains the soliton solutions that we want to obtain.

\section{Reduction of (2.17) to the Ablowitz-Ladik equations}

To explain the idea behind the splitting we are going to do, it seems reasonable to pass from the vertex notation to one based on the shifts/translations $\mathbb{T}$,

$$
f_{A}=f, \quad f_{W}=\mathbb{T}_{W} f, \quad f_{S}=\mathbb{T}_{S} f, \quad \text { etc. }
$$

In this notation, the bilinear system (2.17) can be rewritten as

$$
\begin{aligned}
& 0=\tau^{2}-\rho \sigma-\left(\mathbb{T}_{S} \tau\right)\left(\mathbb{T}_{N} \tau\right) \\
& 0=\tau\left(\mathbb{T}_{L} \sigma\right)-\sigma\left(\mathbb{T}_{L} \tau\right)+\mu\left[\left(\mathbb{T}_{S} \tau\right)\left(\mathbb{T}_{W} \sigma\right)-\left(\mathbb{T}_{S} \sigma\right)\left(\mathbb{T}_{W} \tau\right)\right] \\
& 0=\tau\left(\mathbb{T}_{L} \rho\right)-\rho\left(\mathbb{T}_{L} \tau\right)+\mu\left[\left(\mathbb{T}_{S} \tau\right)\left(\mathbb{T}_{W} \rho\right)-\left(\mathbb{T}_{S} \rho\right)\left(\mathbb{T}_{W} \tau\right)\right] \\
& 0=\sigma\left(\mathbb{T}_{W} \rho\right)+\rho\left(\mathbb{T}_{W} \sigma\right)-\left(\mu+\mu^{-1}\right) \tau\left(\mathbb{T}_{W} \tau\right)+\mu\left(\mathbb{T}_{S} \tau\right)\left(\mathbb{T}_{U} \tau\right)+\mu^{-1}\left(\mathbb{T}_{N} \tau\right)\left(\mathbb{T}_{L} \tau\right)
\end{aligned}
$$

Each point of both direct and dual lattices can be reached by a composition of two basic shifts, say, $\mathbb{T}_{S}$ and $\mathbb{T}_{W}$,

$$
\mathbb{T}_{N}=\mathbb{T}_{S}^{-1}, \quad \mathbb{T}_{L}=\mathbb{T}_{S} \mathbb{T}_{W}, \quad \mathbb{T}_{U}=\mathbb{T}_{S}^{-1} \mathbb{T}_{W}, \quad \text { etc. }
$$

and hence all equations can be presented in terms of these two translations only. The trick that leads to the reduction to three-term equations is to use three shifts as a basic system. One of them is, say, $\mathbb{T}_{S}$ while two more shifts come from the splitting of $\mathbb{T}_{L}$,

$$
\mathbb{T}_{L}=\mathbb{T}_{X} \mathbb{T}_{Y}
$$

or, alternatively, $\mathbb{T}_{S} \mathbb{T}_{W}=\mathbb{T}_{X} \mathbb{T}_{Y}$. Application of this construction to our equations leads to 
Proposition 3.1. A wide range of solutions for the field equations (1.6) and (1.7) can be obtained from the bilinear system of Hirota-like equations

$$
\begin{aligned}
& 0=\tau^{2}-\rho \sigma-\left(\mathbb{T}_{S} \tau\right)\left(\mathbb{T}_{N} \tau\right), \\
& 0=\tau\left(\mathbb{T}_{X} \tau\right)-\sigma\left(\mathbb{T}_{X} \rho\right)-\left(\mathbb{T}_{S} \tau\right)\left(\mathbb{T}_{N X} \tau\right), \\
& 0=\mu\left(\mathbb{T}_{S} \sigma\right)\left(\mathbb{T}_{X} \rho\right)-\left(\mathbb{T}_{S} \tau\right)\left(\mathbb{T}_{X} \tau\right)+\tau\left(\mathbb{T}_{S X} \tau\right), \\
& 0=\tau\left(\mathbb{T}_{Y} \tau\right)-\rho\left(\mathbb{T}_{Y} \sigma\right)-\left(\mathbb{T}_{S} \tau\right)\left(\mathbb{T}_{N Y} \tau\right), \\
& 0=\mu\left(\mathbb{T}_{S} \rho\right)\left(\mathbb{T}_{Y} \sigma\right)-\left(\mathbb{T}_{S} \tau\right)\left(\mathbb{T}_{Y} \tau\right)+\tau\left(\mathbb{T}_{S Y} \tau\right) .
\end{aligned}
$$

One can find a proof of this statement in Appendix A. Geometrically, this constructions can be interpreted as if we considered our plane as a part of three-dimensional lattice and presented our bilinear equations as a projection of more simple three-dimensional system.

Equations (3.2)-(3.6) are closely related to the Ablowitz-Ladik system [1, 2]. Indeed, if we think of the tau-functions as depending on a discrete index, $n$, and two infinite sets of 'times', $\left\{t_{j}\right\}_{j=1}^{\infty}$ and $\left\{\bar{t}_{k}\right\}_{k=1}^{\infty}$, and identify $\mathbb{T}_{S, N}$ with the shifts $n \rightarrow n \pm 1$ and $\mathbb{T}_{X, Y}$ with the Miwa shifts $t_{j} \rightarrow t_{j} \pm i \xi^{j} / j$ and $\bar{t}_{k} \rightarrow \bar{t}_{k} \pm i \eta^{k} / k$, then (3.2)-(3.6), considered as functional equations, describe the positive and negative flows of the ALH (see [24]). Thus, the calculations of the previous section could be replaced with the statement that each solution for the ALH provides a solution for the 2DTHL (not only the soliton ones that we derive below). However, it seems that such approach is not the best in the case of the model we study. In principle, it is possible to rewrite equations (3.2)-(3.6) in terms of $u_{\boldsymbol{r}}$ and $\phi_{\boldsymbol{r}}$, but the resulting equations (which we do not write here) are rather cumbersome and can hardly give clear understanding of, say, the origin of $u-p$ relations (2.11).

\section{Soliton solutions for (3.2)-(3.6)}

In this section we derive the soliton solutions for the bilinear system (3.2)-(3.6). This will be done in two steps. First we solve it without taking into account the condition $r=-q^{*}$ and, secondly, find the restrictions that should be imposed on the parameters of the solutions to meet it.

Since equations (3.2)-(3.6) are nothing but the Ablowitz-Ladik system, we use some of the results of papers $[1,2]$, namely the structure of the soliton solutions without developing the inverse scattering transform from scratch.

The main blocks for constructing the $N$-soliton solutions are the matrices that satisfy the 'almost rank-one' conditions

$$
\mathrm{LA}-\mathrm{AR}=|1\rangle\langle\mathrm{a}|, \quad \mathrm{RB}-\mathrm{BL}=| 1\rangle\langle\mathrm{b}| .
$$

Here $\mathrm{L}$ and $\mathrm{R}$ are constant diagonal matrices, $|1\rangle$ is a constant $N$-component column, $\langle\mathrm{a}|$ and $\langle\mathrm{b}|$ are $N$-component rows depending on the coordinates of the problem.

We look for solutions of the form, similar to the form of soliton solutions for the AblowitzLadik model:

$$
\tau=\operatorname{det}|1-\mathrm{AB}|=\operatorname{det}|1-\mathrm{BA}|
$$

and

$$
q=\left\langle\mathrm{a}\left|\mathrm{FR}^{-1}\right| 1\right\rangle, \quad r=-\left\langle\mathrm{b}\left|\mathrm{L}^{-1} \mathrm{G}\right| 1\right\rangle .
$$

Here 1 is the $N \times N$ unit matrix,

$$
F=(1-B A)^{-1}, \quad G=(1-A B)^{-1}
$$


and $\langle\mathrm{a}|\mathrm{M}| 1\rangle$ is the standard row-matrix-column product: $\langle\mathrm{a}|\mathrm{M}| b\rangle=\sum_{j k} a_{j} M_{j k} b_{k}$, where $a_{j}, M_{j k}$ and $b_{k}$ are components of a row $\langle\mathrm{a}|$, a matrix $\mathrm{M}$ and a column $|b\rangle$.

The second part of the 'solitonic ansatz' is that the action of the shifts $\mathbb{T}_{S, X, Y, \ldots}$ can be implemented as the right multiplication by diagonal constant matrices. The structure of these matrices, that are rational functions of $\mathrm{L}$ and $\mathrm{R}$, can be obtained, again, from [1, 2]. The resulting formulae can be written as follows:

Proposition 4.1. Soliton solutions for (3.2)-(3.6) are given by

$$
\tau=\operatorname{det}|1-\mathrm{AB}|=\operatorname{det}|1-\mathrm{BA}|
$$

and

$$
\sigma=\tau\left\langle\mathrm{a}\left|\mathrm{FR}^{-1}\right| 1\right\rangle, \quad \rho=-\tau\left\langle\mathrm{b}\left|\mathrm{L}^{-1} \mathrm{G}\right| 1\right\rangle
$$

with the following actions of the $\mathbb{T}$-shifts:

$$
\begin{array}{ll}
\mathbb{T}_{S} \mathrm{~A}=\mathrm{AR}, & \mathbb{T}_{S} \mathrm{~B}=\mathrm{BL}^{-1}, \\
\mathbb{T}_{X} \mathrm{~A}=\mathrm{A}(1+\mu \mathrm{R}), & \mathbb{T}_{X} \mathrm{~B}=\mathrm{B}(1+\mu \mathrm{L})^{-1}, \\
\mathbb{T}_{Y} \mathrm{~A}=\mathrm{A}\left(1+\mu \mathrm{R}^{-1}\right)^{-1}, & \mathbb{T}_{Y} \mathrm{~B}=\mathrm{B}\left(1+\mu \mathrm{L}^{-1}\right)
\end{array}
$$

and

$$
\begin{array}{llrl}
\mathbb{T}_{S}\langle\mathrm{a}| & =\langle\mathrm{a}| \mathrm{R}, & & \mathbb{T}_{S}\langle\mathrm{~b}|=\langle\mathrm{b}| \mathrm{L}^{-1}, \\
\mathbb{T}_{X}\langle\mathrm{a}|=\langle\mathrm{a}|(1+\mu \mathrm{R}), & & \mathbb{T}_{X}\langle\mathrm{~b}|=\langle\mathrm{b}|(1+\mu \mathrm{L})^{-1}, \\
\mathbb{T}_{Y}\langle\mathrm{a}|=\langle\mathrm{a}|\left(1+\mu \mathrm{R}^{-1}\right)^{-1}, & & \mathbb{T}_{Y}\langle\mathrm{~b}|=\langle\mathrm{b}|\left(1+\mu \mathrm{L}^{-1}\right) .
\end{array}
$$

This proposition is proved in Appendix B.

After having derived the 'general' soliton solutions for (3.2)-(3.6), we have to ensure the 'physical' involution $r=-q^{*}$, or

$$
\tau^{*}=\tau, \quad \rho=-\sigma^{*}
$$

bearing in mind that

$$
\mu^{*}=\epsilon \mu, \quad \epsilon= \pm 1
$$

which follows from the fact that $\mu^{2}=-J_{2} \xi_{2} / J_{1} \xi_{1}$ is real, but possibly negative, number (see $(2.16))$.

Omitting rather straightforward calculations, we present here the following results: the relationships between the matrices $A$ and $B$, and hence between the rows $\langle a|$ and $\langle b|$, that ensure (4.6) are

$$
\mathrm{L}^{*}=\epsilon \mathrm{R}^{-1}, \quad \mathrm{R}^{*}=\epsilon \mathrm{L}^{-1}
$$

together with

$$
A^{*}=-\mathrm{RBL}^{-1}, \quad \mathrm{~B}^{*}=-\mathrm{LAR}^{-1}
$$

and

$$
\left\langle\mathrm{a}^{*}\right|=\epsilon\langle\mathrm{b}| \mathrm{L}^{-2} .
$$


Restrictions (4.7), (4.8) can be resolved by introducing, instead of A and B, one matrix C,

$$
A=C R, \quad B=-\epsilon R^{-1} C^{*},
$$

and the single row $\langle c|$ instead of $\langle a|$ and $\langle b|$,

$$
\langle\mathrm{a}|=\langle\mathrm{c}| \mathrm{R}, \quad\langle\mathrm{b}|=\left\langle\mathrm{c}^{*}\right| \mathrm{L} .
$$

Definitions (4.9) and (4.10) enable to present F and G as

$$
\mathrm{F}=\mathrm{R}^{-1} \mathrm{HR}, \quad \mathrm{G}=\mathrm{H}^{*},
$$

where

$$
\mathrm{H}=\left(1+\epsilon \mathrm{C}^{*} \mathrm{C}\right)^{-1}
$$

and to rewrite (4.2) and (4.3) as

$$
\tau=\operatorname{det}\left|1+\epsilon \mathrm{C}^{*} \mathrm{C}\right|
$$

and

$$
q=\langle c|\mathrm{H}| 1\rangle, \quad r=-\left\langle\mathrm{c}^{*}\left|\mathrm{H}^{*}\right| 1\right\rangle,
$$

which clearly demonstrates the fulfilment of (4.6).

\section{Solitons of the $2 \mathrm{DTHL}$}

Now we have all necessary to present the $N$-soliton solutions for equations (1.6), (1.7), i.e. to write down the expressions describing the solitons of the 2DTHL.

First we have to return from the next-neighbour notation to the 'absolute' one noting that for any lattice vector,

$$
\boldsymbol{r}=m_{1} \boldsymbol{\delta}_{1}+m_{2} \boldsymbol{\delta}_{2},
$$

and any function $f_{r}$

$$
f_{\boldsymbol{r}}=\mathbb{T}_{R}^{m_{1}} \mathbb{T}_{U}^{m_{2}} f
$$

where $f$ is the value of the function at some fixed point. Recalling that $\mathbb{T}_{L}=\mathbb{T}_{X} \mathbb{T}_{Y}$ and that

$$
\mathbb{T}_{R}=\mathbb{T}_{L}^{-1}, \quad \mathbb{T}_{U}=\mathbb{T}_{L} \mathbb{T}_{S}^{-2}
$$

one can introduce the matrices $\mathrm{C}_{\boldsymbol{r}}$ and the rows $\left\langle\mathrm{c}_{\boldsymbol{r}}\right|$ by

$$
\mathrm{C}_{\boldsymbol{r}}=\mathrm{CM}_{1}^{m_{1}} \mathrm{M}_{2}^{m_{2}}, \quad\left\langle\mathrm{c}_{\boldsymbol{r}}\right|=\langle\mathrm{c}| \mathrm{M}_{1}^{m_{1}} \mathrm{M}_{2}^{m_{2}},
$$

where, as follows from (4.4),

$$
\mathrm{M}_{1}=\left(1+\mu \mathrm{R}^{-1}\right)(1+\mu \mathrm{R})^{-1}, \quad \mathrm{M}_{2}=\left(\mathrm{R}^{-1}+\mu 1\right)(\mathrm{R}+\mu 1)^{-1}
$$

with constant $\mathrm{C}$ and $\langle\mathrm{c}|$ related by

$$
\mathrm{LC}-\mathrm{CR}=|1\rangle\langle\mathrm{c}| \quad \text { or } \quad(\mathrm{C})_{j k}=\frac{c_{k}}{L_{j}-R_{k}}=\frac{\epsilon R_{j}^{*} c_{k}}{1-\epsilon R_{j}^{*} R_{k}} .
$$


The parameter $\mu$ depends on vector $\boldsymbol{k}$ that defines the asymptotics of solutions (see (2.14)),

$$
\mu=\mu(\boldsymbol{k})=\sqrt{-\frac{J_{2}}{J_{1}}} \exp \left[\frac{1}{2}\left(\boldsymbol{k}, \boldsymbol{\delta}_{1}-\boldsymbol{\delta}_{2}\right)\right]
$$

while the constant $\epsilon$ is given by

$$
\epsilon=-\operatorname{sign} J_{1} J_{2} \text {. }
$$

In this notation, the main formulae of the previous section become

$$
q_{r}=\left\langle c_{r}\left|\mathrm{H}_{r}\right| 1\right\rangle
$$

where

$$
\mathrm{H}_{\boldsymbol{r}}=\left(1+\epsilon \mathrm{C}_{r}^{*} \mathrm{C}_{\boldsymbol{r}}\right)^{-1} \quad \text { and } \quad \tau_{\boldsymbol{r}}=\operatorname{det}\left|1+\epsilon \mathrm{C}_{r}^{*} \mathrm{C}_{\boldsymbol{r}}\right| .
$$

Finally, one can present the soliton solutions of the model considered in this paper as follows: the vectors $\phi_{\boldsymbol{r}}$ are given by

$$
\phi_{\boldsymbol{r}}=\left(\begin{array}{c}
\sin \theta_{\boldsymbol{r}} \cos \varphi_{\boldsymbol{r}} \\
\sin \theta_{\boldsymbol{r}} \sin \varphi_{\boldsymbol{r}} \\
\cos \theta_{\boldsymbol{r}}
\end{array}\right)
$$

with

$$
\theta_{\boldsymbol{r}}=\arcsin \frac{\left|q_{\boldsymbol{r}}\right|}{1+\left|q_{\boldsymbol{r}}\right|^{2}}, \quad \varphi_{\boldsymbol{r}}=\arg q_{\boldsymbol{r}},
$$

where $q_{\boldsymbol{r}}$ are defined by (5.1) while

$$
u_{\boldsymbol{r}}=(\boldsymbol{k}, \boldsymbol{r})+\frac{1}{2} \ln \frac{\tau_{\boldsymbol{r}}^{+}}{\tau_{\boldsymbol{r}}^{-}}
$$

with

$$
\tau_{\boldsymbol{r}}^{ \pm}=\mathbb{T}_{S}^{ \pm 1} \tau_{\boldsymbol{r}}=\operatorname{det}\left|1+\epsilon \mathrm{C}_{\boldsymbol{r}}^{*} \mathrm{~L}^{\mp 1} \mathrm{C}_{\boldsymbol{r}} \mathrm{R}^{ \pm 1}\right|
$$

\section{Conclusion}

In this paper, we have presented the nonlinear $2 \mathrm{D}$ lattice and have obtained its $N$-soliton solutions. To conclude, we would like to give some comments related to the proposed model, the method we used to solve it and to outline the possible continuation of this work.

The key moment in the bilinearization of the field equations were equations (2.6) and (2.7). Since we did not expect to obtain the general solution and the objective was do derive some particular ones, we have not studied it in details. We used it as an ansatz, a trick that helps us to achieve our goal (even despite the loss of the generality). However this substitution, nonlocal and rather cumbersome when rewritten in terms of the original variables, $u_{\boldsymbol{r}}$ and $\phi_{\boldsymbol{r}}$, surely needs to be studied in a more detailed way. In some sense, equations (2.6) and (2.7) can be viewed as an quadrilateral version of the star-triangle transformation (see [3] and references therein). To our opinion, it may prove useful in the studies of other two-dimensional lattice models.

Considering the loss of generality, we have to admit that our approach, is surely a reduction. First, the $u-\boldsymbol{\phi}$ 'mixing' that we made by introducing the tau-functions by (2.1), (2.2) and (2.12) 
narrows the class of solutions that we can obtain. For example, the 'frozen spin' configurations $\phi_{\boldsymbol{r}}=(0,0,1)^{T}$ are described by the Hirota-like model (1.3) whose solutions hardly can be obtained in the framework of the method of this paper because the restriction $\phi_{A}=(0,0,1)^{T}$ implies $q_{A}=0$ and $p_{A}=1$ which drastically simplifies all equations from Propositions 2.1 and 3.1 leavig us with almost trivial solutions for the model (1.3). A similar effect occurs when we split the second-order equations into the first-order system (see section 3), which can be viewed as the second step of the reduction. To illustrate this fact we would like to note, for example, that model (1.2) considered in this paper admits a non-trivial one-dimensional reduction $u_{\boldsymbol{r}}=$ $u_{\boldsymbol{r}+\boldsymbol{\delta}_{1}}, \phi_{\boldsymbol{r}}=\phi_{\boldsymbol{r}+\boldsymbol{\delta}_{1}}$ whose most interesting solutions, again, cannot obtained directly from the ones derived above: one can easily see from equations (3.2)-(3.6) that the reduction $\mathbb{T}_{L} u=u$, $\mathbb{T}_{L} \phi=\phi$ leads to $\mathbb{T}_{S} \tau=$ const $\cdot \tau$ which means that equations from Proposition 3.1 provide only almost trivial solutions for the one-dimensional problem. To summarize, not all solutions for (1.6) and (1.7), can be obtained from (3.2)-(3.6). However the class of solutions described by (3.2)-(3.6) is rather rich and seems to include, not strictly speaking, almost all essentially two-dimensional solutions that can be written explicitly.

As it was said above, equations (3.2)-(3.6) belong to the ALH. The fact that ALH-equations lead to various Toda-like ones is not new. For example, it has been shown in [25] that equations describing the famous two-dimensional Toda lattice can be splitted into ones for the simplest ALH flows. Second example is the recent paper [23], where the author discusses the relationships between the ALH and the relativistic Toda [5, 22] and the two-dimensional Volterra [17] models. On the other hand, there is a number of papers that demonstrate the links between the ALH and Heisenberg-like models. Probably the first such example is Ishimori classical spin chain [16] which is gauge equivalent to the discrete nonlinear Schrödinger equation (one of the most wellstudied equations of the ALH). Another example one can find in [21], where the authors study a model of Landau-Lifshitz fields interacting in a Heisenberg-like way. However there is an important distinction between the present and the above-cited works. The case is that all models from [5, 16, 17, 21, 22, 23, 25] are chains. Indeed, even the two-dimensional Toda and Volterra models are two-dimensional in the sense that they are equations for the functions of two continuous but only one discrete variables, while the 2DTHL is two-dimensional as a lattice. Thus, this paper is an attempt to extend the 'reducing to the ALH' approach to a family of 2D models whose most elegant examples are Hirota models [13, 14] (though they are usually considered in the framework of discrete-time evolution and not as the 2D lattices).

The fact that the 2DTHL possesses the $N$-soliton solutions is a strong evidence of the integrability of the problem $[7,8,9,10,15,19]$ (see also $[11,12]$ for its application to discrete systems). However, the situations with the 2DTHL can be more complicated: there is a possibility that equations from Propositions 2.1 and 3.1 belong to the family of the so-called 'conditionally integrable' systems introduced by Dorizzi et al. [6]. In any case, the questions related to the integrability of the 2DTHL, its zero-curvature representation and conserved quantities surely deserve further studies.

Another feasible continuation of this work is related to prospective applications to the theory of magnetic systems. Traditionally, the soliton solutions are most representative ones for any integrable system. However, from the viewpoint of the theory of magnetism, it would be interesting to find other families of solutions, except the non-topological solitons presented in this paper, that correspond to more typical magnetic structures as, e.g., domain walls and finite magnetic domains.

\section{A Proof of Proposition 3.1}

The proof of Proposition 3.1 is straightforward and consists in presenting the right-hand sides of the equations from Proposition 2.1 as combinations of the right-hand sides of the equations 
from Proposition 3.1. To this end, consider the following definitions:

$$
\begin{aligned}
& \mathfrak{a}=\tau^{2}-\rho \sigma-\left(\mathbb{T}_{S} \tau\right)\left(\mathbb{T}_{N} \tau\right), \\
& \mathfrak{b}^{(1)}=\tau\left(\mathbb{T}_{X} \tau\right)-\sigma\left(\mathbb{T}_{X} \rho\right)-\left(\mathbb{T}_{S} \tau\right)\left(\mathbb{T}_{N X} \tau\right), \\
& \mathfrak{b}^{(2)}=\mu\left(\mathbb{T}_{S} \sigma\right)\left(\mathbb{T}_{X} \rho\right)-\left(\mathbb{T}_{S} \tau\right)\left(\mathbb{T}_{X} \tau\right)+\tau\left(\mathbb{T}_{S X} \tau\right), \\
& \mathfrak{c}^{(1)}=\tau\left(\mathbb{T}_{Y} \tau\right)-\rho\left(\mathbb{T}_{Y} \sigma\right)-\left(\mathbb{T}_{S} \tau\right)\left(\mathbb{T}_{N Y} \tau\right), \\
& \mathfrak{c}^{(2)}=\mu\left(\mathbb{T}_{S} \rho\right)\left(\mathbb{T}_{Y} \sigma\right)-\left(\mathbb{T}_{S} \tau\right)\left(\mathbb{T}_{Y} \tau\right)+\tau\left(\mathbb{T}_{S Y} \tau\right)
\end{aligned}
$$

(which are shortcuts for the right-hand sides of (3.2)-(3.6)). Our aim is to prove that vanishing of $\mathfrak{a}, \mathfrak{b}^{(1,2)}$ and $\mathfrak{c}^{(1,2)}$ implies vanishing of

$$
\begin{aligned}
& \mathfrak{Q}=\tau\left(\mathbb{T}_{L} \sigma\right)-\sigma\left(\mathbb{T}_{L} \tau\right)+\mu\left(\mathbb{T}_{S} \tau\right)\left(\mathbb{T}_{W} \sigma\right)-\mu\left(\mathbb{T}_{S} \sigma\right)\left(\mathbb{T}_{W} \tau\right) \\
& \mathfrak{R}=\tau\left(\mathbb{T}_{L} \rho\right)-\rho\left(\mathbb{T}_{L} \tau\right)+\mu\left(\mathbb{T}_{S} \tau\right)\left(\mathbb{T}_{W} \rho\right)-\mu\left(\mathbb{T}_{S} \rho\right)\left(\mathbb{T}_{W} \tau\right) \\
& \mathfrak{P}=\sigma\left(\mathbb{T}_{W} \rho\right)+\rho\left(\mathbb{T}_{W} \sigma\right)-\left(\mu+\mu^{-1}\right) \tau\left(\mathbb{T}_{W} \tau\right)+\mu\left(\mathbb{T}_{S} \tau\right)\left(\mathbb{T}_{U} \tau\right)+\mu^{-1}\left(\mathbb{T}_{N} \tau\right)\left(\mathbb{T}_{L} \tau\right)
\end{aligned}
$$

(the right-hand sides of equations (3.1)). The first part of the proof is simple: it can be shown that $\mathfrak{Q}$ and $\mathfrak{R}$ are linear combinations of $\mathfrak{b}^{(1,2)}$ and $\mathfrak{c}^{(1,2)}$. Indeed, one can check that

$$
\begin{aligned}
& \left(\mathbb{T}_{X} \rho\right) \mathfrak{Q}=\left(\mathbb{T}_{L} \tau\right) \mathfrak{b}^{(1)}-\left(\mathbb{T}_{W} \tau\right) \mathfrak{b}^{(2)}-\tau\left(\mathbb{T}_{X} \mathfrak{c}^{(1)}\right)+\left(\mathbb{T}_{S} \tau\right)\left(\mathbb{T}_{N X} \mathfrak{c}^{(2)}\right) \\
& \left(\mathbb{T}_{Y} \sigma\right) \mathfrak{R}=-\tau\left(\mathbb{T}_{Y} \mathfrak{b}^{(1)}\right)+\left(\mathbb{T}_{S} \tau\right)\left(\mathbb{T}_{N Y} \mathfrak{b}^{(2)}\right)+\left(\mathbb{T}_{L} \tau\right) \mathfrak{c}^{(1)}-\left(\mathbb{T}_{W} \tau\right) \mathfrak{c}^{(2)}
\end{aligned}
$$

Thus

$$
\mathfrak{b}^{(1,2)}=\mathfrak{c}^{(1,2)}=0 \quad \Rightarrow \quad \mathfrak{Q}=\mathfrak{R}=0 .
$$

As to $\mathfrak{P}$, the calculations are slightly more complicated and can be performed in two steps. First, writing the system composed of (A.1) and shifted (A.2),

$$
\begin{aligned}
& \mathfrak{b}^{(1)}=\tau\left(\mathbb{T}_{X} \tau\right)-\sigma\left(\mathbb{T}_{X} \rho\right)-\left(\mathbb{T}_{S} \tau\right)\left(\mathbb{T}_{N X} \tau\right), \\
& \mathbb{T}_{N X} \mathfrak{c}^{(2)}=\mu\left(\mathbb{T}_{W} \sigma\right)\left(\mathbb{T}_{X} \rho\right)-\left(\mathbb{T}_{W} \tau\right)\left(\mathbb{T}_{X} \tau\right)+\left(\mathbb{T}_{L} \tau\right)\left(\mathbb{T}_{N X} \tau\right),
\end{aligned}
$$

and eliminating $\left(\mathbb{T}_{X} \rho\right)$ one arrives at

$$
\sigma\left(\mathbb{T}_{N X} \mathfrak{c}^{(2)}\right)+\mu\left(\mathbb{T}_{W} \sigma\right) \mathfrak{b}^{(1)}=A\left(\mathbb{T}_{X} \tau\right)-B\left(\mathbb{T}_{N X} \tau\right),
$$

where

$$
A=\mu \tau\left(\mathbb{T}_{W} \sigma\right)-\sigma\left(\mathbb{T}_{W} \tau\right), \quad B=\mu\left(\mathbb{T}_{S} \tau\right)\left(\mathbb{T}_{W} \sigma\right)-\sigma\left(\mathbb{T}_{L} \tau\right) .
$$

In a similar way, elimination of $\mathbb{T}_{N X} \rho$ from

$$
\begin{aligned}
& \mathbb{T}_{N} \mathfrak{b}^{(2)}=\mu \sigma\left(\mathbb{T}_{N X} \rho\right)-\tau\left(\mathbb{T}_{N X} \tau\right)+\left(\mathbb{T}_{N} \tau\right)\left(\mathbb{T}_{X} \tau\right), \\
& \mathbb{T}_{N X} \mathfrak{c}^{(1)}=\left(\mathbb{T}_{W} \tau\right)\left(\mathbb{T}_{N X} \tau\right)-\left(\mathbb{T}_{W} \sigma\right)\left(\mathbb{T}_{N X} \rho\right)-\left(\mathbb{T}_{U} \tau\right)\left(\mathbb{T}_{X} \tau\right)
\end{aligned}
$$

leads to

$$
\left(\mathbb{T}_{W} \sigma\right)\left(\mathbb{T}_{N} \mathfrak{b}^{(2)}\right)+\mu \sigma\left(\mathbb{T}_{N X} \mathfrak{c}^{(1)}\right)=-C\left(\mathbb{T}_{X} \tau\right)+D\left(\mathbb{T}_{N X} \tau\right)
$$

with

$$
C=\mu \sigma\left(\mathbb{T}_{U} \tau\right)-\left(\mathbb{T}_{N} \tau\right)\left(\mathbb{T}_{W} \sigma\right), \quad D=\mu \sigma\left(\mathbb{T}_{W} \tau\right)-\tau\left(\mathbb{T}_{W} \sigma\right)
$$


or, in the matrix form,

$$
\left(\begin{array}{cc}
A & -B \\
-C & D
\end{array}\right)\left(\begin{array}{c}
\mathbb{T}_{X} \tau \\
\mathbb{T}_{N X} \tau
\end{array}\right)=\left(\begin{array}{l}
\mathfrak{d}^{(1)} \\
\mathfrak{d}^{(2)}
\end{array}\right)
$$

where $\mathfrak{d}^{(1,2)}$ are linear combinations of shifted $\mathfrak{b}^{(1,2)}$ and $\mathfrak{c}^{(1,2)}$. On the other hand, one can straightforwardly verify the identity

$$
\sigma\left(\mathbb{T}_{W} \sigma\right) \mathfrak{P}+\mu^{-1}(A D-B C)+\left(\mathbb{T}_{W} \sigma\right)^{2} \mathfrak{a}+\sigma^{2}\left(\mathbb{T}_{W} \mathfrak{a}\right)=0 .
$$

Equations (3.1) imply vanishing of the last two terms of the left-hand side of the last equation and of the vector $\left(\mathfrak{d}^{(1)}, \mathfrak{d}^{(2)}\right)^{T}$, which means that the determinant of the matrix that appears in the left-hand side of (A.3) is zero, $A D-B C=0$, since we assume $\tau \neq 0$ (and, hence, $\mathbb{T}_{X} \tau \neq 0$ and $\mathbb{T}_{N X} \tau \neq 0$ ). This, together with (A.4), leads to

$$
\mathfrak{a}=\mathfrak{b}^{(1,2)}=\mathfrak{c}^{(1,2)}=0 \quad \Rightarrow \quad \mathfrak{P}=0
$$

which completes the proof of the fact that equations (3.2)-(3.6) imply (3.1) which, in its turn, completes the proof of Proposition 3.1.

\section{B Verification of the solitonic ansatz}

To make the following formulae more readable we use here the 'node' notation not only for the nodes of lattices (direct and dual) but for the results of the auxiliary shifts $\mathbb{T}_{X, Y}$ as well:

$$
f_{X}=\mathbb{T}_{X} f, \quad f_{Y}=\mathbb{T}_{Y} f, \quad f_{S X}=\mathbb{T}_{X} f_{S}=\mathbb{T}_{X} \mathbb{T}_{S} f, \quad \text { etc. }
$$

First let us prove the fact that equations (4.3)-(4.5) imply (3.3). Applying (4.1) and the $S$-part of (4.4) to the product $\mathrm{B}_{S} \mathrm{~A}_{S}$ one can get

$$
\mathrm{BA}-\mathrm{B}_{S} \mathrm{~A}_{S}=\mathrm{B}_{S}|1\rangle\langle\mathrm{a}|
$$

which leads to

$$
\mathrm{FF}_{S}^{-1}=1+\mathrm{FB}_{S}|1\rangle\langle\mathrm{a}|
$$

and

$$
\mathrm{F}_{S} \mathrm{~F}^{-1}=1-\mathrm{F}_{S} \mathrm{~B}_{S}|1\rangle\langle\mathrm{a}| .
$$

Shifting this equation in the $\mathbb{T}_{N} \mathbb{T}_{X}$-direction one can obtain

$$
\mathrm{KF}_{X} \mathrm{~F}_{N X}^{-1} \mathrm{~K}^{-1}=1-\mathrm{KF}_{X} \mathrm{~B}_{X}|1\rangle\langle\mathrm{a}|
$$

where

$$
\mathrm{K}=\mathrm{R}^{-1}+\mu
$$

(we have used the fact that $\left\langle\mathrm{a}_{N X}\right| \mathrm{K}^{-1}=\langle\mathrm{a}|$ ). Multiplying (B.1) and (B.2) one arrives at

$$
\mathrm{FF}_{S}^{-1} \mathrm{KF}_{X} \mathrm{~F}_{N X}^{-1} \mathrm{~K}^{-1}=1+\mathrm{FU}|1\rangle\langle\mathrm{a}| \text {, }
$$

where

$$
\mathrm{U}=\mathrm{B}_{S}-\mathrm{F}_{S}^{-1} \mathrm{KF}_{X} \mathrm{~B}_{X}=\mathrm{B}_{S}\left(1+\mathrm{A}_{S} \mathrm{KF}_{X} \mathrm{~B}_{X}\right)-\mathrm{KF}_{X} \mathrm{~B}_{X}
$$


Noting that $\mathrm{A}_{S} \mathrm{~K}=\mathrm{A}_{X}$ and applying the identities

$$
\mathrm{FB}=\mathrm{BG}, \quad 1+\mathrm{AFB}=\mathrm{G}
$$

one can continue the calculations as follows:

$$
\mathrm{U}=\mathrm{B}_{S}\left(1+\mathrm{A}_{X} \mathrm{~F}_{X} \mathrm{~B}_{X}\right)-\mathrm{KF}_{X} \mathrm{~B}_{X}=\left(\mathrm{B}_{S}-\mathrm{KB} \mathrm{B}_{X}\right) \mathrm{G}_{X}=\mathrm{R}^{-1}|1\rangle\left\langle\mathrm{b}_{S X}\right| \mathrm{G}_{X}
$$

After substitution of $\mathrm{U}$ in (B.3) and calculating the determinants of the both sides one arrives at

$$
\frac{\tau_{S} \tau_{N X}}{\tau \tau_{X}}=1-q r_{X}
$$

(here, the identities $\tau=1 / \operatorname{det} \mathbf{F}$ and $\operatorname{det}(1+|\mathbf{u}\rangle\langle\mathbf{v}|)=1+\langle\mathrm{v} \mid \mathrm{u}\rangle$ have been used) which at the level of the tau-functions is

$$
0=\tau \tau_{X}-\sigma \rho_{X}-\tau_{S} \tau_{N X}
$$

Thus we have proved that our solutions satisfy equations (3.3).

Noting that the implementation of the shift $\mathbb{T}_{X}$ depends analytically on $\mu$ and $\left.\mathbb{T}_{X}\right|_{\mu=0}$ is the unit operator, one obtains from the above calculations, by sending $\mu \rightarrow 0$, that our ansatz ensures (3.2) as well.

In a similar way, applying (4.1) to $\mathrm{B}_{X} \mathrm{~A}_{X}$ one can obtain

$$
\mathrm{F}_{X} \mathrm{~F}^{-1}=1-\mu \mathrm{F}_{X} \mathrm{~B}_{X}|1\rangle\left\langle\mathrm{a}\left|, \quad \mathrm{FF}_{X}^{-1}=1+\mu \mathrm{FB}_{X}\right| 1\right\rangle\langle\mathrm{a}|
$$

and then

$$
\mathrm{F}_{S} \mathrm{~F}_{S X}^{-1} \mathrm{R}^{-1} \mathrm{~F}_{X} \mathrm{~F}^{-1} \mathrm{R}=1+\mu \mathrm{F}_{S} \mathrm{~V}|1\rangle\left\langle\mathrm{a}_{S}\right|
$$

where

$$
\begin{aligned}
\mathrm{V} & =\mathrm{B}_{S X}-\mathrm{F}_{S X}^{-1} \mathrm{R}^{-1} \mathrm{~F}_{X} \mathrm{~B}_{X}=\mathrm{B}_{S X}\left(1+\mathrm{A}_{X} \mathrm{~F}_{X} \mathrm{~B}_{X}\right)-\mathrm{R}^{-1} \mathrm{~F}_{X} \mathrm{~B}_{X} \\
& =\left(\mathrm{B}_{S X}-\mathrm{R}^{-1} \mathrm{~B}_{X}\right) \mathrm{G}_{X}=\mathrm{R}^{-1}|1\rangle\left\langle\mathrm{b}_{S X}\right| \mathrm{G}_{X},
\end{aligned}
$$

which leads to

$$
\frac{\tau \tau_{S X}}{\tau_{S} \tau_{X}}=1-\mu q_{S} r_{X}
$$

and

$$
0=\mu \sigma_{S} \rho_{X}-\tau_{S} \tau_{X}+\tau \tau_{S X}
$$

This proves the fact that our solutions satisfy (3.4).

Considering the $\mathbb{T}_{Y}$-equations, we do not present here the calculations similar to ones discussed above, leaving the verification of (3.5) and (3.6) to the reader.

\section{Acknowledgements}

We would like to thank the referees for careful reading the manuscript and for providing useful comments and suggestions which helped us to improve the paper. 


\section{References}

[1] Ablowitz M.J., Ladik J.F., Nonlinear differential-difference equations, J. Math. Phys. 16 (1975), 598-603.

[2] Ablowitz M.J., Ladik J.F., Nonlinear differential-difference equations and Fourier analysis, J. Math. Phys. 17 (1976), 1011-1018.

[3] Baxter R.J., Exactly solved models in statistical mechanics, Academic Press Inc., London, 1982.

[4] Brown H.A., Luttinger J.M., Ferromagnetic and antiferromagnetic Curie temperatures, Phys. Rev. 100 (1955), 685-692.

[5] Bruschi M., Ragnisco O., Lax representation and complete integrability for the periodic relativistic Toda lattice, Phys. Lett. A 134 (1989), 365-370.

[6] Dorizzi B., Grammaticos B., Ramani A., Winternitz P., Are all the equations of the Kadomtsev-Petviashvili hierarchy integrable?, J. Math. Phys. 27 (1986), 2848-2852.

[7] Hietarinta J., A search for bilinear equations passing Hirota's three-soliton condition. I. KdV-type bilinear equations, J. Math. Phys. 28 (1987), 1732-1742.

[8] Hietarinta J., A search for bilinear equations passing Hirota's three-soliton condition. II. mKdV-type bilinear equations, J. Math. Phys. 28 (1987), 2094-2101.

[9] Hietarinta J., A search for bilinear equations passing Hirota's three-soliton condition. III. Sine-Gordon-type bilinear equations, J. Math. Phys. 28 (1987), 2586-2592.

[10] Hietarinta J., A search for bilinear equations passing Hirota's three-soliton condition. IV. Complex bilinear equations, J. Math. Phys. 29 (1988), 628-635.

[11] Hietarinta J., Zhang D.J., Hirota's method and the search for integrable partial difference equations. 1. Equations on a $3 \times 3$ stencil, J. Difference Equ. Appl., to appear, arXiv:1210.4708.

[12] Hietarinta J., Zhang D.J., Hirota's method and the search for integrable partial difference equations. 2. Equations on a $2 \times N$ stencil, in Report of RIAM Symposium No. 22AO-S8 "Development in Nonlinear Wave: Phenomena and Modeling", Research Institute for Applied Mechanics, Kyushu University, 2011, 30-36.

[13] Hirota R., Nonlinear partial difference equations. I. A difference analogue of the Korteweg-de Vries equation, J. Phys. Soc. Japan 43 (1977), 1424-1433.

[14] Hirota R., Nonlinear partial difference equations. II. Discrete-time Toda equation, J. Phys. Soc. Japan 43 (1977), 2074-2078.

[15] Hirota R., The direct method in soliton theory, Cambridge Tracts in Mathematics, Vol. 155, Cambridge University Press, Cambridge, 2004.

[16] Ishimori Y., An integrable classical spin chain, J. Phys. Soc. Japan 51 (1982), 3417-3418.

[17] Leznov A.N., Saveliev M.V., Smirnov V.G., Explicit solutions to two-dimensionalized Volterra equations, Lett. Math. Phys. 4 (1980), 445-449.

[18] Mattis D.C., The theory of magnetism. I. Statics and dynamics, Springer Series in Solid-State Sciences, Vol. 17, Springer-Verlag, Berlin, 1981.

[19] Newell A.C., Yunbo Z., The Hirota conditions, J. Math. Phys. 27 (1986), 2016-2021.

[20] Papageorgiou V., Grammaticos B., Ramani A., Orthogonal polynomial approach to discrete Lax pairs for initial-boundary value problems of the QD algorithm, Lett. Math. Phys. 34 (1995), 91-101.

[21] Pritula G.M., Vekslerchik V.E., Toda-Heisenberg chain: interacting $\sigma$-fields in two dimensions, J. Nonlinear Math. Phys. 18 (2011), 443-459, arXiv:1108.5937.

[22] Ruijsenaars S.N.M., Relativistic Toda systems, Comm. Math. Phys. 133 (1990), 217-247.

[23] Vekslerchik V.E., Explicit solutions for a $(2+1)$-dimensional Toda-like chain, J. Phys. A: Math. Theor. 46 (2013), 055202, 22 pages, arXiv:1301.0414.

[24] Vekslerchik V.E., Functional representation of the Ablowitz-Ladik hierarchy. II, J. Nonlinear Math. Phys. 9 (2002), 157-180, solv-int/9812020.

[25] Vekslerchik V.E., The 2D Toda lattice and the Ablowitz-Ladik hierarchy, Inverse Problems 11 (1995), 463-479. 